\title{
Interacting hepatic PAI-1/tPA gene regulatory pathways influence impaired fibrinolysis severity in obesity
}

\author{
Ze Zheng, ${ }^{1}$ Keiko Nakamura, ${ }^{1,2,3}$ Shana Gershbaum, ${ }^{1,4}$ Xiaobo Wang, ${ }^{1}$ Sherry Thomas, ${ }^{1}$ Marc Bessler, ${ }^{5}$ Beth Schrope, ${ }^{5}$ \\ Abraham Krikhely, ${ }^{5}$ Rui-Ming Liu, ${ }^{6}$ Lale Ozcan, ${ }^{1}$ José A. López, ${ }^{7,8}$ and Ira Tabas ${ }^{1,9,10}$ \\ 'Department of Medicine, Columbia University Irving Medical Center, New York, New York, USA. ${ }^{2}$ Graduate School of Medicine and ${ }^{3}$ Faculty of Medicine, University of Tokyo, Tokyo, Japan. ${ }^{4}$ Neuroscience and \\ Behavior Department, Barnard College, New York, New York, USA. ${ }^{5}$ Department of Surgery, Columbia University Irving Medical Center, New York, New York, USA. ${ }^{6}$ Division of Pulmonary Allergy, and Critical \\ Care Medicine, Department of Medicine, University of Alabama at Birmingham, Birmingham, Alabama, USA. `Department of Medicine, University of Washington, Seattle, Washington, USA. ${ }^{8}$ Bloodworks \\ Research Institute, Seattle, Washington, USA. ${ }^{9}$ Department of Physiology and ${ }^{10}$ Department of Pathology and Cell Biology, Columbia University Irving Medical Center, New York, New York, USA.
}

\begin{abstract}
Fibrinolysis is initiated by tissue-type plasminogen activator (tPA) and inhibited by plasminogen activator inhibitor 1 (PAI-1). In obese humans, plasma PAI-1 and tPA proteins are increased, but PAI-1 dominates, leading to reduced fibrinolysis and thrombosis. To understand tPA-PAI-1 regulation in obesity, we focused on hepatocytes, a functionally important source of tPA and PAI-1 that sense obesity-induced metabolic stress. We showed that obese mice, like humans, had reduced fibrinolysis and increased plasma PAI-1 and tPA, due largely to their increased hepatocyte expression. A decrease in the PAI-1 (SERPINE1) gene corepressor Rev-Erbo increased PAI-1, which then increased the tPA gene PLAT via a PAI-1/LRP1/PKA/p-CREB1 pathway. This pathway was partially counterbalanced by increased DACH1, a PLAT-negative regulator. We focused on the PAI-1/PLAT pathway, which mitigates the reduction in fibrinolysis in obesity. Thus, silencing hepatocyte PAI-1, CREB1, or tPA in obese mice lowered plasma TPA and further impaired fibrinolysis. The PAI-1/PLAT pathway was present in primary human hepatocytes, and associations among PAI-1, tPA, and PLAT in livers from obese and lean humans were consistent with these findings. Knowledge of PAI-1 and tPA regulation in hepatocytes in obesity may suggest therapeutic strategies for improving fibrinolysis and lowering the risk of thrombosis in this setting.
\end{abstract}

\section{Introduction}

Obesity, which has now reached epidemic proportions, increases risks for arterial, venous, and microvascular thrombosis, including coronary thrombosis $(1,2)$, stroke $(3)$, deep vein thrombosis $(4,5)$, pulmonary embolism $(5,6)$, and thrombotic thrombocytopenic purpura $(5,7)$. A major contributor to increased thrombosis in obesity is a reduction of fibrinolysis $(8,9)$, the primary mechanism that dissolves a blood clot (10). Tissue-type plasminogen activator (tPA) initiates fibrinolysis by converting the zymogen plasminogen to plasmin, a serine protease that degrades the fibrin clot $(11,12)$. We have shown in lean mice that hepatocytes maintain a basal level of circulating tPA that influences fibrinolysis following vessel injury (13). As a serine protease, tPA is inhibited primarily by its serpin plasminogen activator inhibitor 1 (PAI1) (10). In obesity, plasminogen activator activity and fibrinolysis in blood are reduced due to an increase in PAI-1 $(9,14)$. Coincidentally, tPA is also increased in obesity $(15,16)$, which may represent a compensatory response. These observations raise a number of critical unanswered questions, namely, what are the sources and regulatory mechanisms controlling PAI-1 and tPA in

Conflict of interest: The authors have declared that no conflict of interest exists. Copyright: () 2020, Zheng et al. This is an open access article published under the terms of the Creative Commons Attribution 4.0 International License.

Submitted: December 20, 2019; Accepted: May 13, 2020; Published: July 13, 2020

Reference information: J Clin Invest. 2020;130(8):4348-4359.

https://doi.org/10.1172/JCl135919. obesity, and does the tPA response partially compensate for the impairment in fibrinolysis caused by high PAI- 1 in obesity?

One source of PAI- 1 is adipocytes, and elevated adipocyte PAI-1 has been proposed as the dominant source for increased PAI-1 and the consequent fibrinolysis defect in obesity $(9,14,15)$. Arguing against this idea is the observation that when blood from subcutaneous adipose arteries and veins was assayed for PAI-1 protein and activity in obese humans, there was no PAI-1 arteriovenous gradient (17). These data raise the possibility that one or more other sources of circulating PAI-1 may be important in obesity. Hepatocytes are sensitive to the metabolic stress that occurs in obesity and, as is the case with tPA (13), hepatocytes can produce PAI-1 (18-21). However, the regulation of hepatocyte PAI-1 in obesity and whether an increase in hepatocyte-derived PAI-1 contributes to the fibrinolysis defect in obesity are not known. These gaps of knowledge also exist for hepatocyte tPA in obesity. In this context, we now show that hepatocytes are a substantial source of both plasma tPA and PAI-1 in mice with diet-induced obesity. The increase in hepatocyte PAI-1 in obesity is linked to a decrease in the PAI-1 (SERPINE1) gene corepressor Rev-Erb $\alpha$. Moreover, reducing hepatocyte PAI-1 in obese mice blocked the increase in both liver tPA and functionally active plasma tPA, indicating the existence of a hepatocyte PAI-1/tPA pathway in obesity. Although this tPA response pathway is partially counteracted by a coexisting tPA-suppressive pathway mediated by the corepressor DACH1, we provide evidence that the PAI-1/ tPA pathway limits the degree of impairment of fibrinolysis in 


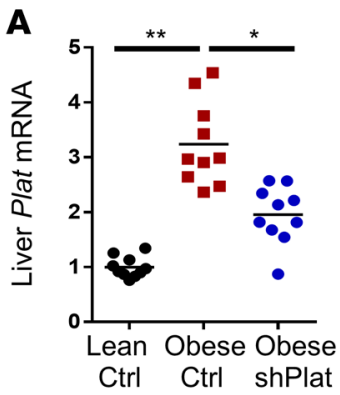

C

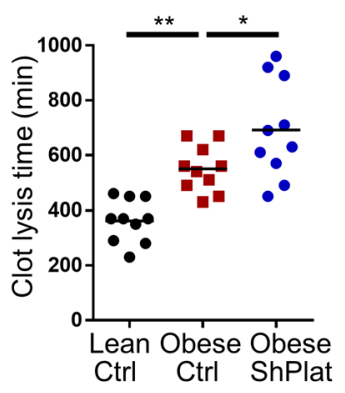

F Obese Serpine $1^{f / / t}$ mice
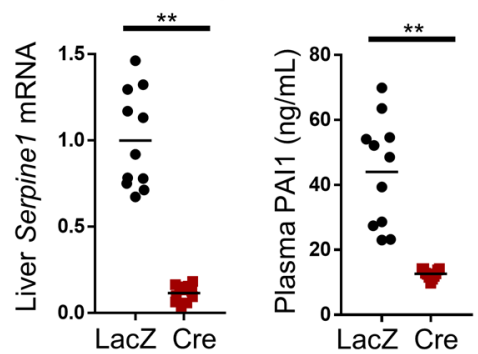

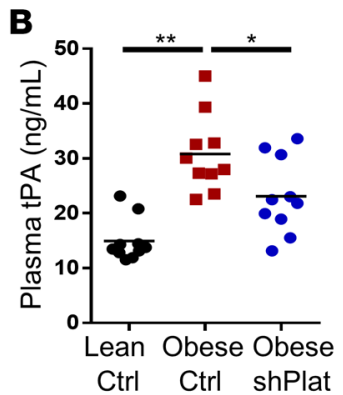

D

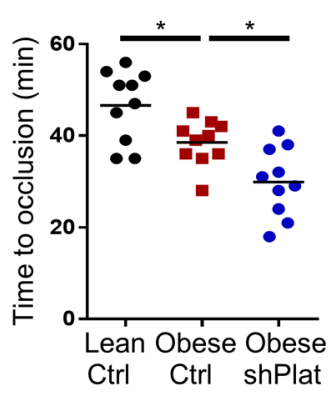

G

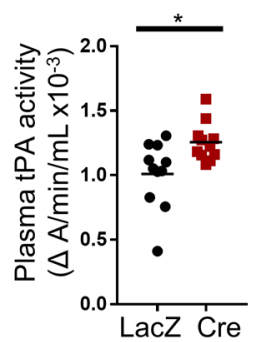

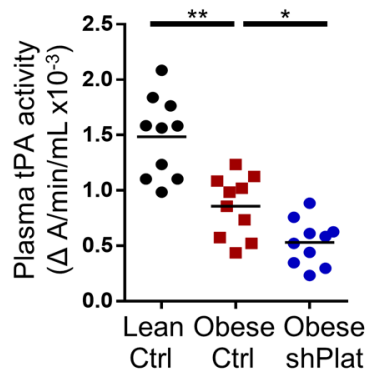

E Human liver and adipose tissue

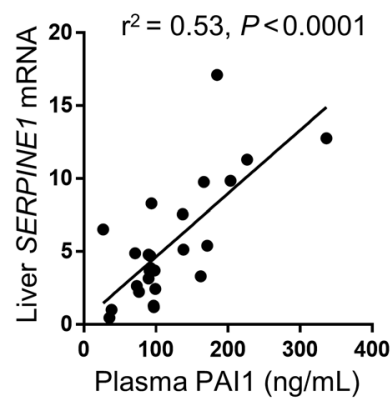

H

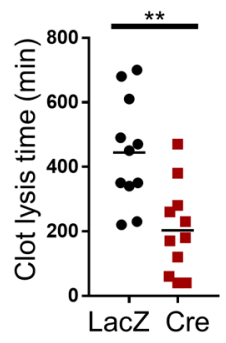

I

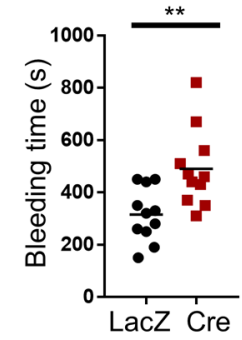

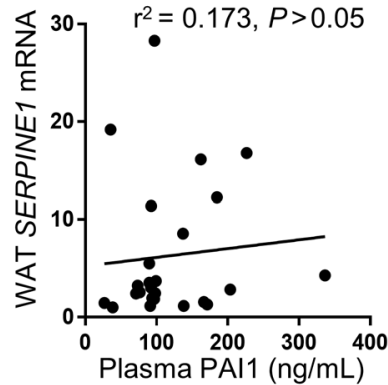

Figure 1. Hepatocyte tPA is increased in DIO mice, but a larger increase in hepatocyte PAI-1 causes a net impairment of fibrinolysis. (A-C) Chow-fed mice (Lean) or DIO mice were injected intravenously with AAV8-H1-shPlat (shPlat) to silence the tPA gene Plat in hepatocytes or with control AAV8-H1-Scr (Ctrl). After 4 weeks, the following parameters were measured: (A) liver Plat mRNA; (B) plasma tPA protein concentration and plasma tPA activity; (C) fibrinolytic activity measured by euglobulin clot-lysis assay; and (D) time to occlusive carotid arterial thrombosis induced by rose bengal/laser photochemical injury. Horizontal lines in the dot-density plots indicate mean values. $n=10$ mice/group. ${ }^{*} P<0.05$; ${ }^{* *} P<0.01$, 1-way ANOVA followed by Tukey's test. (E) Samples of human liver and omental white adipose tissue (WAT) from the subjects described in Supplemental Table 1 were assayed for SERPINE1 mRNA, and the plasma from these subjects was assayed for PAI-1 concentration. The graph shows plots of the indicated correlations, which were analyzed by linear regression, with the $r^{2}$ and $P$ values indicated in the graph. (F-I) Serpine $f^{f / f I}$ mice were fed a high-fat diet for 4 months and then injected intravenously with AAV8-TBG-Cre (Cre) to target the PAI-1 gene Seprine1 in hepatocytes or with control AAV8-TBC-LacZ (LacZ). After 4 weeks, the following parameters were measured: (F) liver Serpine1 mRNA and plasma PAI-1 protein concentration; (C) plasma tPA activity measured by enzymatic assay; (H) plasma fibrinolytic activity measured by the euglobulin clot-lysis assay; and (I) tail-bleeding time and time to occlusive carotid arterial thrombosis induced by rose bengal/ laser photochemical injury. $n=11$ mice/group. ${ }^{*} P<0.05 ;{ }^{* *} P<0.01,2$-tailed Student's $t$ test.

obesity. Accordingly, the fibrinolysis defect in obesity becomes even worse when the PAI-1/tPA pathway is genetically targeted in mice. The mechanism of the PAI-1/tPA pathway involves induction of the tPA gene PLAT through a pathway in which PAI1, through its receptor LRP1, activates the PLAT inducer cAMPresponsive element-binding protein 1 (CREB1). The PAI-1/PLAT pathway is present in primary human hepatocytes, and livers, but not adipose tissue, from obese and lean humans showed associations among DACH1, tPA, and PLAT that are consistent with these findings. These findings add insight into the regulation of fibrinolysis in obesity, thereby suggesting new strategies for lowering thrombotic risk in this condition.

\section{Results}

Hepatocyte tPA is increased in diet-induced obese mice, but a larger increase in hepatocyte PAI-1 causes a net impairment of fibrinolysis. Plasma tPA protein concentration was increased in obese versus lean mice, but plasma tPA activity was reduced, plasma clot-lysis time was delayed, and time to occlusive carotid arterial thrombosis induced by photochemical injury was shortened in obese mice (Supplemental Figure 1A; supplemental material available online with this article; https://doi.org/10.1172/JCI135919DS1). These findings are consistent with data in obese humans (22-25). Based on previous reports $(16,24,26,27)$, we reasoned that reduced plasma tPA activity despite increased tPA protein could be explained 
A Obese Dach1 $1^{t / f t}$ mice
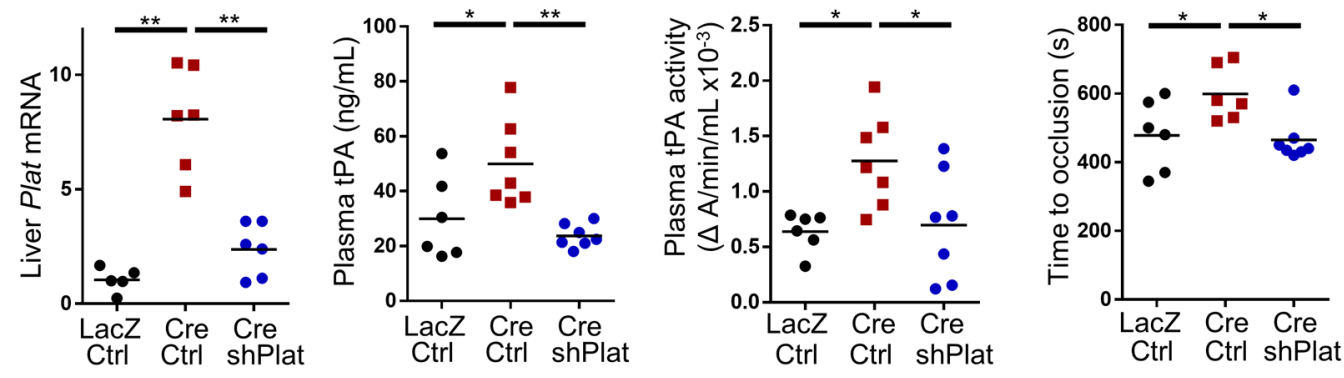

B Human subjects
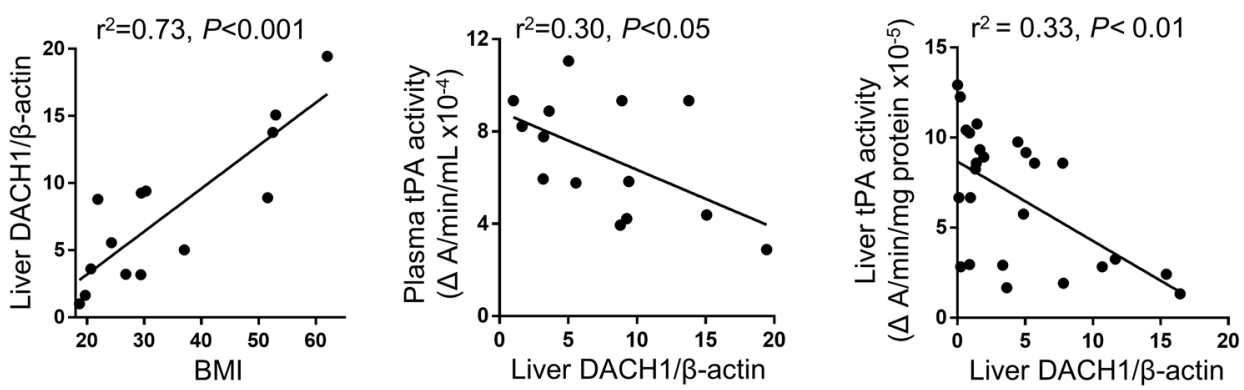

Figure 2. Increased hepatocyte DACH1 in obesity limits the rise in tPA and thereby contributes to impaired fibrinolysis. (A) Dach $7^{f l / f l}$ mice were fed a high-fat diet for 4 months and then injected intravenously with AAV8-TBG-Cre to target the Plat corepressor Dach1 in hepatocytes or with control AAV8-TBG-LacZ. Mice were also injected with AAV8 control virus or AAV8-H1-shPlat, as indicated. After 4 weeks, the following parameters were measured: liver Plat mRNA, plasma tPA protein concentration, plasma tPA activity, and time to occlusive carotid arterial thrombosis induced by rose bengal/laser photochemical injury. Horizontal lines in the dot-density plots indicate mean values. $n=5-7$ mice/group. ${ }^{*} P<0.05$; ${ }^{* *} P<0.01$, 1-way ANOVA followed by Tukey's test. (B) For the left and middle graphs, liver specimens from 14 human subjects with a wide range of BMI, as described previously (22), were assayed for DACH1/ $\beta$-actin densitometric ratio on immunoblot, as shown in Supplemental Figure 3B, and samples of their plasma were assayed for tPA activity. For the right graph, liver specimens from 25 human subjects, as previously described (13), were assayed for liver DACH1/ $\beta$-actin ratio (13) and tPA activity. Graphs show plots of the indicated correlations, which were analyzed by linear regression, with $r^{2}$ and $P$ values indicated in the graph.

by increased plasma PAI-1. Consistent with this formulation, plasma PAI-1 was markedly increased in the plasma of obese mice (Supplemental Figure 1B).

To elucidate the cellular source of increased tPA and PAI-1 expression in obesity, we focused on hepatocytes because hepatocytes are profoundly affected by obesity and, in lean mice, hepatocytes contribute substantially to the basal level of plasma tPA and to fibrinolysis in response to vessel injury (13). Beginning with tPA, we found that Plat mRNA was increased in the livers of obese versus lean mice, although tPA activity in the liver was lower (Supplemental Figure 1C). Consistent with our previous observation (13), hepatic and endothelial Plat mRNA levels were comparable in lean mice, but only hepatic Plat mRNA was increased by obesity (Supplemental Figure 1D). To test the functional significance of the increase in liver tPA in obesity, we silenced hepatocyte tPA in obese mice using hepatocyte-specific shRNA (AAV8-H1-shPlat) (13), titrating the dosage of the virus to lower hepatic Plat close to the level in lean mice, but not lower (Figure 1A). This level of silencing ( $40 \%)$ led to an approximately $25 \%$ decrease in plasma tPA protein, an approximately $32 \%$ decrease in plasma tPA activity, an approximately $26 \%$ increase in plasma clot-lysis time, and an approximately $22 \%$ decrease in postinjury carotid arteryocclusion time (Figure 1, B-D), all without changing plasma levels of PAI- 1 protein and $\alpha 2$-antiplasmin (A2AP), the major inhibitor of plasmin (Supplemental Figure 1, E and F). These data demonstrate the functional significance of increased hepatocyte tPA in obesity, i.e., the defect in fibrinolysis in obesity is even greater when this increase is prevented. Thus, the obesity-induced increase in hepatocyte tPA limits the fibrinolytic impairment in obesity.

We next turned our attention to PAI-1 and its mRNA, Serpine1. We found markedly increased expression of Serpine1 mRNA in the livers of obese versus lean mice (Supplemental Figure 1G). Although PAI-1 protein levels were increased in both liver and omental white adipose tissue in obese versus lean mice, liver PAI-1 protein was higher than adipose PAI-1 protein (Supplemental Figure $1 \mathrm{H}$ ). In human specimens, plasma PAI-1 protein was strongly correlated with liver SERPINE1 mRNA level, but not with SERPINE1 mRNA, in adipose tissue (Figure 1E and Supplemental Table 1). Most importantly, specific targeting of hepatocyte PAI-1 in obese Serpine $1^{f l / f l}$ mice (28) using AAV8-TBG-Cre, which silences floxed genes exclusively in hepatocytes $(13,29-32)$, decreased plasma PAI-1 protein approximately $70 \%$, increased plasma tPA activity, shortened clot-lysis time, and increased both tail-bleeding time and time to occlusive thrombosis (Figure 1, F-I), all without changing plasma A2AP levels (Supplemental Figure 1I). In summary, obesity is associated with increased expression of tPA and PAI- 1 in hepatocytes, both of which influence plasma fibrinolysis. Although the dominant effect is impaired fibrinolysis secondary to increased hepatocyte PAI-1, the increase in hepatocyte tPA limits the degree of this impairment. 
A Obese Serpine $1^{\text {th/t }}$ mice
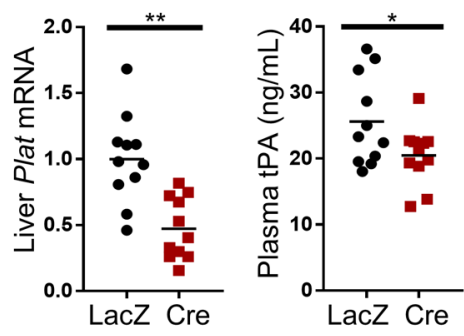

C Primary human hepatocytes

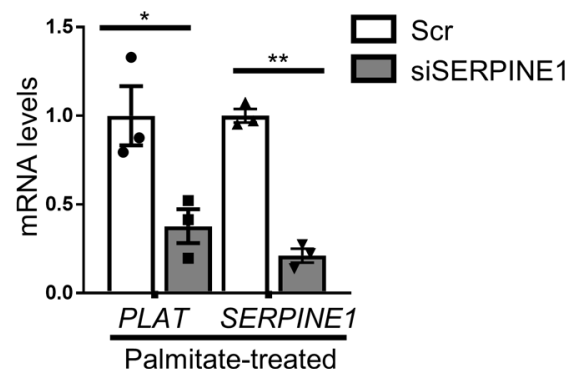

B
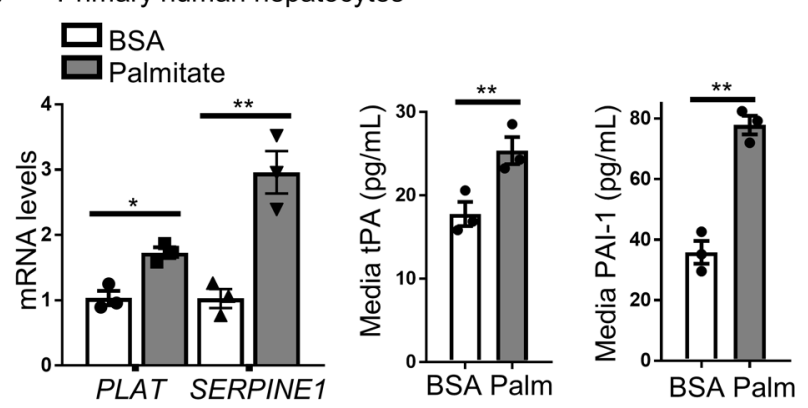

D Human liver

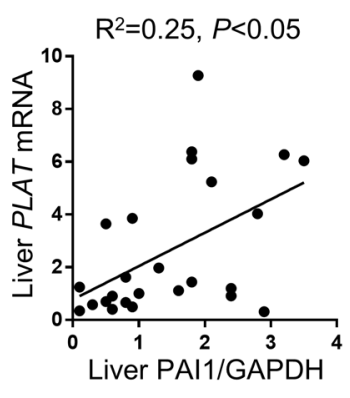

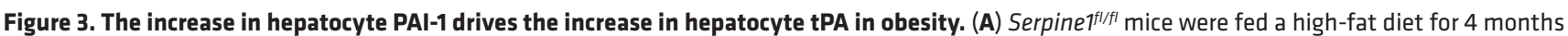
and then injected intravenously with either AAV8-TBG-Cre or control AAV8-TBG-LacZ. After 4 weeks, liver Plat mRNA and plasma tPA protein concentrations were measured. Horizontal lines in the dot-density plots indicate mean values. $n=11$ mice/group. ${ }^{*} P<0.05 ;{ }^{*} P<0.01,2$-tailed Student's $t$ test. (B) Human primary hepatocytes were treated for 16 hours with $100 \mu \mathrm{M}$ palmitate (PALM) in BSA solution, or BSA solution control. The cells were assayed for PLAT and SERPINE1 mRNA, and the media were assayed for TPA and PAI-1 protein concentration. (C) Human primary hepatocytes were transfected with siSERPINE1 or scrambled control (Scr). After 24 hours, cells were treated with $100 \mu \mathrm{M}$ palmitate for an additional 16 hours and then assayed for PLAT and SERPINE1 mRNA. In B and C, results are shown as mean \pm SEM. $n=3$ sets of cells/group. ${ }^{*} P<0.05 ;{ }^{*} P<0.01$, 2-tailed Student's $t$ test. (D) Liver specimens from the subjects described in Supplemental Table 1 were assayed for PAI-1/GAPDH densitometric ratio by immunoblot, as shown in Supplemental Figure 3C, and for PLAT mRNA. These data were then subjected to correlation analysis, as described in the legend for Figure 2B.

Increased hepatocyte DACH1 in obesity limits the rise in $T P A$ and thereby contributes to impaired fibrinolysis. The expression of hepatocyte $P L A T$ is negatively regulated in lean mice by the corepressor DACH1, which represses the PLAT inducer ATF6 (13). We reasoned that this pathway would be particularly relevant to obesity, as hepatocyte DACH1 is increased in the livers of obese mice and humans (32), which we verified here (Supplemental Figure 2A). To test this idea, we injected diet-induced obese (DIO) Dach $1^{f / f f l}$ mice with AAV8-TBG-Cre, which targets DACH1 exclusively in hepatocytes (13), or with control AAV8-TBG-LacZ (Supplemental Figure 2B). Consistent with the hypothesis, targeting hepatocyte DACH1 (HC-DACH1-KO mice) increased liver Plat mRNA, plasma tPA concentration and activity, and time to occlusive carotid thrombosis (Figure 2A). To determine whether the increased fibrinolytic activity was due to increased hepatic tPA expression, we silenced hepatocyte Plat in the HC-DACH1-KO mice using AAV8-H1-sh-tPA to the level seen in control AAV8-TBG-LacZ-treated obese mice. This intervention normalized all of the fibrinolytic parameters in the obese HC-DACH1-KO mice (Figure 2A). Neither deleting hepatocyte DACH1 nor treating these mice with shPlat changed plasma PAI-1 concentration (Supplemental Figure 2C). Finally, we looked for correlations among BMI, liver DACH1, liver tPA activity, and plasma tPA activity in humans. Liver DACH1 levels rose with increasing BMI, as shown in our previous study (13) and quanti- fied here, and TPA activities in liver and plasma were negatively correlated with liver DACH1 (Figure 2B and Supplemental Table 2). Similar correlations were observed in mice with a wide range in body weight (Supplemental Figure 2D). In summary, the increase in hepatocyte tPA in obesity is limited by the counteracting process of DACH1-mediated Plat repression, and the resulting net level of hepatic tPA expression is not high enough to overcome PAI-1-mediated impaired fibrinolysis.

The increase in hepatocyte PAI-1 drives the increase in hepatocyte tPA in obesity. Having elucidated a factor that lowers hepatocyte tPA in obesity, i.e., the increase in DACH1, we next sought to understand the counteracting mechanism that causes the net increase in hepatocyte and plasma tPA in obesity. In this context, we noted that liver Plat mRNA and plasma tPA protein were lower in HC-PAI-1$\mathrm{KO}$ than in control obese mice (Figure $3 \mathrm{~A}$ ). These data raised the possibility that PAI-1 induces IPA in hepatocytes in obesity, perhaps representing a compensatory response. We tested this possibility in an in vitro hepatocyte model by incubating primary human hepatocytes with palmitate, which we and others have found can recapitulate certain aspects of hepatocyte biology seen in obesity $(32,33)$. This treatment increased both PLAT and SERPINE1 mRNAs and protein in culture medium (Figure $3 \mathrm{~B}$ ), thus mimicking the response in livers and plasma of obese mice. Most importantly, silencing SERPINE1 in these cells markedly lowered hepatocyte PLAT mRNA 
A

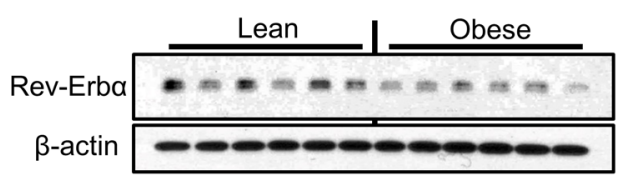

C

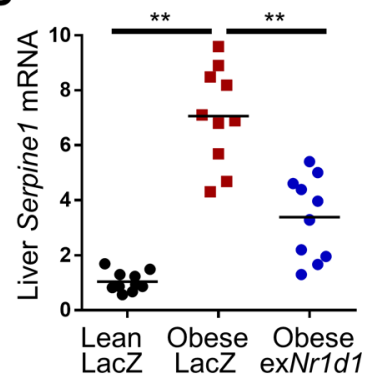

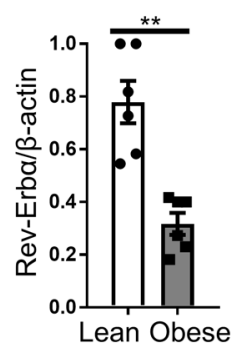

B

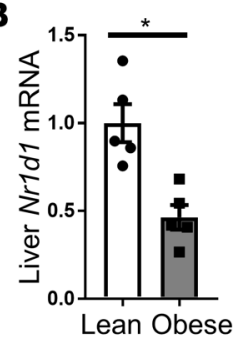

D

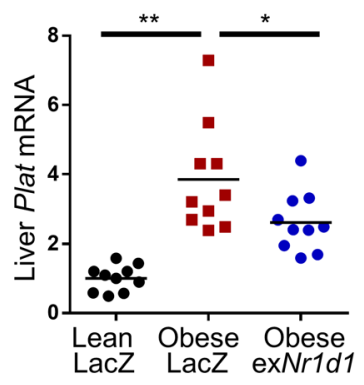

Primary human hepatocytes
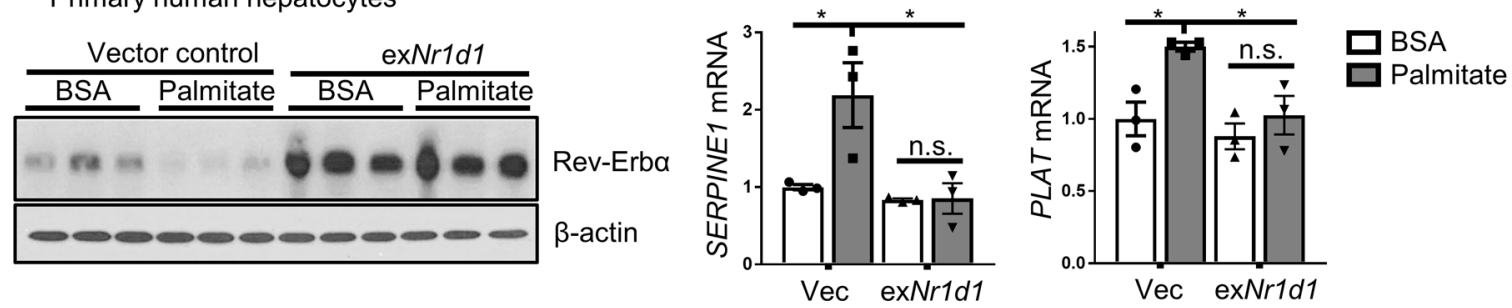

Figure 4. Decreased Rev-Erb $\alpha$ in the livers of obese mice and in palmitate-treated primary human hepatocytes elevates both PAI-1 and tPA expression. (A and B) Livers of lean and DIO mice were assayed for Rev-Erb $\alpha$ protein and $\beta$-actin loading control by immunoblot, with densitometric quantification shown, and for Nr1d1 mRNA. $n=5-6$ mice/group. Data are represented as mean \pm SEM. ${ }^{*} P<0.05$; ${ }^{* *} P<0.01$, 2-tailed Student's $t$ test. (C) Lean and obese mice were injected intravenously with AAV8-TBG-Nr1d1 to increase Rev-Erb $\alpha$ expression in hepatocytes or with AAV8-TBG-LacZ control, as indicated. After 4 weeks, liver Serpine1 and Plat mRNA levels were measured. Horizontal lines in dot-density plots indicate mean values. $n=10$ mice/group. ${ }^{*} P<0.05 ;{ }^{* *} P$ $<0.01$, 1-way ANOVA followed by Tukey's test. (D) Human primary hepatocytes were transfected with a plasmid expressing Nr1d1 to increase expression of Rev-Erba; transfection with empty vector (Vec) served as the control. After 24 hours, cells were treated with $100 \mu \mathrm{M}$ palmitate for 16 hours and then assayed for Rev-Erb $\alpha$ protein by immunoblot and for SERPINE1 and PLAT mRNA by quantitative PCR (qPCR). $n=3$ sets of cells/group. Data are represented as mean \pm SEM. ${ }^{*} P<0.05$, 1-way ANOVA followed by Tukey's test.

(Figure 3C). We obtained similar results with primary mouse hepatocytes (Supplemental Figure 3, A and B). Next, we examined PAI-1 protein and PLAT mRNA in 25 human liver specimens that had a wide range of PAI-1 protein expression (Supplemental Figure $3 \mathrm{C}$ and Supplemental Table 2). The data show a statistically significant correlation between PAI-1 and PLAT in the livers of these subjects (Figure 3D), consistent with the findings in mice and primary human hepatocytes. Together, these data provide evidence for a cell-autonomous PAI-1/PLAT pathway in hepatocytes.

To seek further evidence for this pathway in obesity, we used an alternative strategy to limit endogenous hepatic PAI-1 expression. Rev-Erb $\alpha$ is a transcriptional repressor of PAI-1, and it was shown to be lower in adipose tissue of obese mice than in lean mice $(34,35)$. We found that Rev-Erb $\alpha$ protein and mRNA (Nr1d1) were lower in the livers of obese versus lean mice (Figure 4, A and B). Accordingly, we asked whether restoring Rev-Erb $\alpha$ in hepatocytes in obese mice would lower PAI-1 and thereby lower Plat expression via the new pathway revealed here. For this purpose, obese mice were injected with AAV8-TBG-Nr1d1, which increased liver Nr1d1 and Rev-Erba to levels closer to those of lean mice (Supplemental Figure 4A), or control AAV8-TBG-LacZ. As predicted from prior reports, increasing hepatocyte Rev-Erba in obese mice lowered liver Serpine1, and most importantly, it also lowered liver Plat (Figure 4C). Consistent with hepatocytes being an important source of circulating PAI- 1 and with the dominant effect of hepatocyte PAI- 1 on fibrinolysis, the net effect of hepatocyte Rev-Erb $\alpha$ restoration in obese mice was lower plasma PAI-1, increased plasma tPA activity, and shorter time to clot lysis (Supplemental Figure 4B). These links among Rev-Erba, SERPINE1, and PLAT are cell autonomous, as demonstrated with palmitate-treated primary human hepatocytes: palmitate lowered Rev-Erb $\alpha$, and restoration of Rev-Erb $\alpha$ in these cells lowered both SERPINE1 and PLAT mRNA (Figure 4D). Further, silencing RevErba in primary human hepatocytes using siNR1D1 increased PLAT, and this effect was dependent on the ability of siNR1D1 to increase PAI-1, as simultaneous silencing of PAI-1 using siSERPINE1 prevented the PLAT-raising effect of siNR1D1 (Supplemental Figure 4C). These combined data reveal the presence of a hepatocyte PAI-1/Plat pathway in obesity. Although the effect of PAI-1 is dominant over that of tPA, the presence of this pathway suggests that fibrinolysis would be even more impaired in obesity if this pathway were not present. 
A

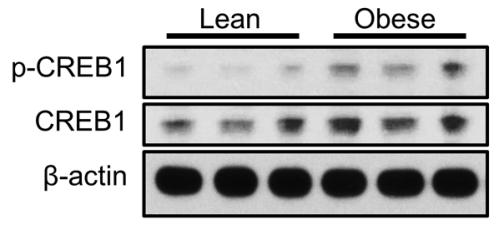

B

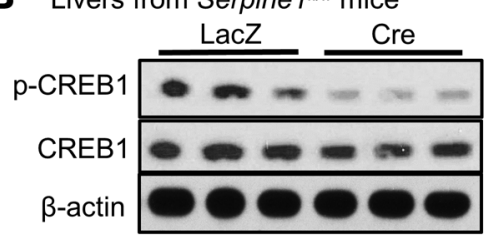

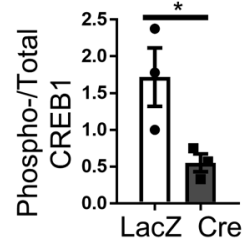

C Palmitate-treated primary human hepatocytes

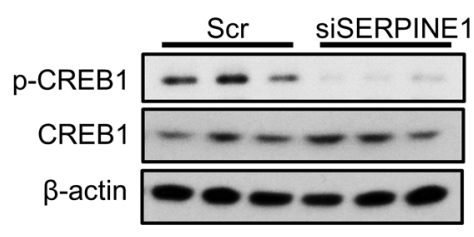

E

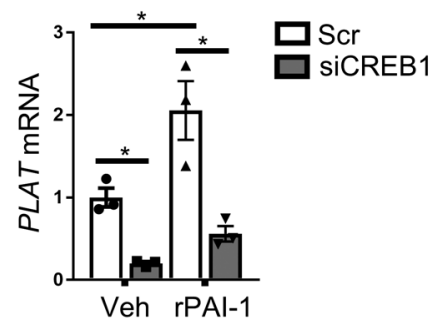

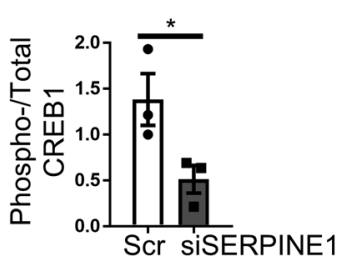

F

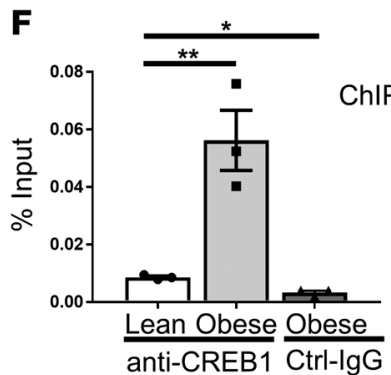

D Primary human hepatocytes

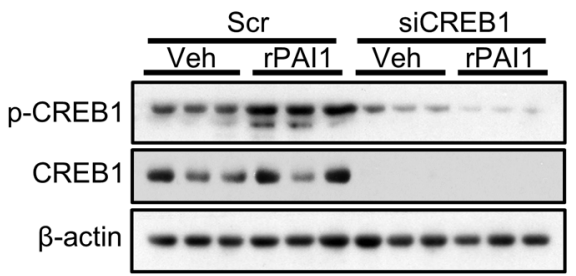

Figure 5. PAI-1 induces CREB1 phosphorylation, which then induces tPA expression in hepatocytes. (A) Livers of lean and obese mice were assayed for p-CREB1 and total CREB1 by immunoblot ( $n=3$ mice/group). (B) Serpine $7^{f l / f l}$ mice were fed a high-fat diet for 4 months and then injected intravenously with either AAV8-TBG-Cre or control AAV8-TBG-LacZ. After 4 weeks, livers were assayed for p-CREB1 and total CREB1 by immunoblot, with densitometric quantification shown. Data are shown as mean \pm SEM. ${ }^{*} P<0.05$, 2-tailed Student's $t$ test. (C) Human primary hepatocytes were transfected with siSERPINE1 or scrambled control. After 24 hours, cells were treated with $100 \mu \mathrm{M}$ palmitate for 16 hours, followed by assay of phosphorylated and total CREB1 by immunoblot, with densitometric quantification shown. Data are represented as mean \pm SEM. ${ }^{*} P<0.05$, 2-tailed Student's $t$ test. (D and $\mathbf{E}$ ) Human primary hepatocytes were transfected with siCREB1 or scrambled control. After 24 hours, cells were treated for 8 hours with $1 \mu \mathrm{g} \mathrm{rPAl}-1 / \mathrm{mL}$ culture medium or vehicle control (Veh). Cells were then assayed for phosphorylated and total CREB1 by immunoblot and for PLAT mRNA by qPCR. $n=3$ sets of cells/group. Data are represented as mean \pm SEM. ${ }^{*} P<0.05$, 1-way ANOVA followed by Tukey's test. (F) Nuclear extracts from the livers of lean or obese mice were subjected to ChIP assay using anti-CREB1 or control lgG (Ctrl-IgC). The proximal promoter region containing the CREB1-binding sequence in the Plat gene was amplified by qPCR and normalized to the values obtained from input DNA. $n=3$ mice/group. Data are represented as mean $\pm \mathrm{SEM}$. ${ }^{*} P<0.05 ;{ }^{* *} P<0.01,1$-way ANOVA followed by Tukey's test.

PAI-1 induces hepatic tPA expression through a LRP1/p-CREB1 pathway. To explore the mechanism whereby PAI-1 induces tPA, we first considered the possibility that PAI-1 reduces DACH1. However, liver DACH1 protein was not affected by deletion of hepatocyte PAI-1 (Supplemental Figure 5A). We next considered the possibility that PAI-1 increases CREB1, which is a transcriptional activator of the PLAT gene in human endothelial cells (36). Although the role of CREB1 in PLAT expression in hepatocytes is not known, CREB1 is expressed in hepatocytes (37). We first compared the livers of lean and obese mice and found that both total and activated (phosphorylated) CREB1 (p-CREB1) were increased in obesity (Figure 5A). Next, we showed that genetic deletion or silencing of hepatocyte PAI-1 decreased p-CREB1 in the livers of obese mice (Figure 5B) and in palmitate-treated primary human hepatocytes (Figure $5 \mathrm{C}$ ). To further establish that PAI-1 activates CREB1 in hepatocytes, we showed that incubation of primary human hepatocytes with recombinant PAI-1 (rPAI1) increased p-CREB1 (Figure 5D). Most importantly, rPAI-1 increased PLAT expression in these cells, and we found that both basal PLAT and rPAI-1-induced PLAT were inhibited by silencing CREB1 (Figure 5E and Supplemental Figure 5B). We obtained similar results using primary mouse hepatocytes (Supplemental Figure 5C). As predicted, ChIP assays in mouse liver revealed a 5 -fold enrichment of CREB1 at the consensus site in the Plat gene promoter in obese versus lean liver, but not in the nonspecific $R p l p O$ promoter (Figure $5 \mathrm{~F}$ and Supplemental Figure 5D). These data support the existence of a PAI-1/CREB1/PLAT pathway in hepatocytes that, owing to the increase in hepatocyte PAI-1 in obesity, is activated in obese liver.

To examine the in vivo relevance of this pathway, we silenced hepatic CREB1 in obese mice by treating DIO CREB1 $1^{f l / f}$ mice with AAV8-TBG-Cre (HC-CREB1-KO) (Figure 6A). Consistent with the hypothesized pathway, targeting hepatocyte CREB1 lowered liver Plat mRNA (Figure 6B). Importantly, this decrease in liver Plat mRNA was associated with decreased plasma tPA protein and plasma tPA activity, increased clot-lysis time, and decreased time to occlusion after carotid injury (Figure 6, C and D), all without a change in plasma PAI-1 (Supplemental Figure 5E). 
A Livers from obese CREB1flifl mice

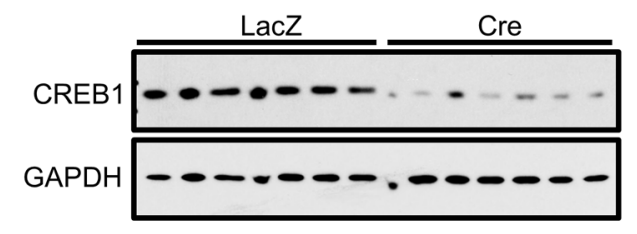

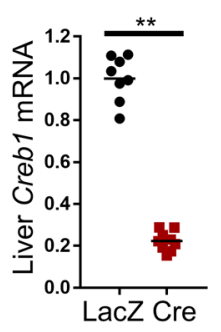

B Obese CREB1 $1^{\text {fllfl }}$ mice

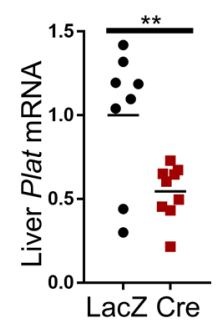

C Obese CREB1 $1^{\text {fl/fl}}$ mice

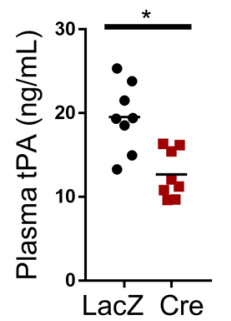

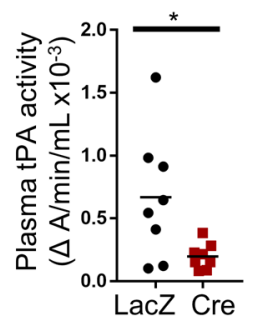

D Obese CREB1 ${ }^{1 / f+1}$ mice

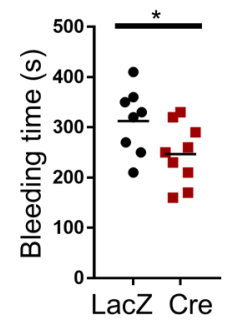

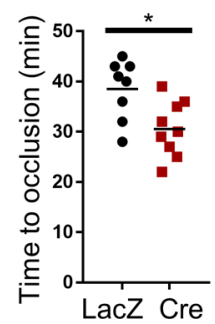

Figure 6. Obese mice with hepatocyte CREB1 deficiency have decreased plasma tPA protein concentration and activity, delayed clot-lysis time, and shortened time to arterial thrombotic occlusion. Creb $1^{f l / f l}$ mice were fed a high-fat diet for 4 months and then injected intravenously with AAV8-TBG-Cre to target Creb1 in hepatocytes or with control AAV8-TBG-LacZ. After 4 weeks, the following parameters were assayed: (A and B) liver CREB1 by immunoblot and liver Creb1 and Plat mRNA by qPCR; (C) plasma tPA protein concentration, plasma tPA activity, and plasma fibrinolytic activity measured by the euglobulin clot-lysis assay; (D) tail-bleeding time and time to occlusive carotid arterial thrombosis induced by rose bengal/laser photochemical injury. $n=$ 7-9 mice/group. ${ }^{*} P<0.05$; ${ }^{* *} P<0.01,2$-tailed Student's $t$ test.

Ligation of LDL receptor-related protein 1 (LRP1), the major cellular receptor for PAI-1, can increase intracellular cAMP levels and the activity of protein kinase A (PKA) (38), the kinase that phosphorylates and activates CREB1 (39). Further, LRP1 has been shown to stimulate CREB1 transcription activity in neurons (40) and adipocytes (41). As LRP1 is abundant on the surface of hepatocytes, we hypothesized that PAI-1 activates CREB1 through LRP1-PKA-mediated signaling to increase tPA expression in obesity. Consistent with this idea, silencing LRP1 in primary human hepatocytes suppressed the rPAI-1-mediated increases in p-CREB1 and PLAT mRNA (Figure 7A). Further, treating these cells with a mutant form of rPAI-1 lacking the LRP1-interacting heparin-binding domain markedly reduced CREB1 phosphorylation and PLAT expression compared with WT rPAI-1 (Figure 7B). We then tested this point in vivo by injecting lean mice with WT rPAI-1, which we predicted would "mimic" the pathway seen in obese liver, or with LRP1-binding mutant rPAI-1, which should be inactive. The total plasma PAI-1 concentration in both WT- and mutant rPAI-1-injected mice reached levels similar to those seen in obese mice (Supplemental Figure 5F compared with Supplemental Figure 1B). WT rPAI-1 increased p-CREB and liver Plat, whereas mutant rPAI-1 did not (Figure 7C). With regard to the role of PKA (above), we found that rPAI-1 induction of p-CREB and PLAT in primary human hepatocytes was prevented by treating the cells with the PKA inhibitor H89 (Figure 7D). Together, these data provide support for a pathway in which the increase in hepatocyte PAI-1 in obesity, mediated at least in part by suppression of Rev-Erb $\alpha$, activates an LRP1/PKA/p-CREB/PLAT pathway that lessens the magnitude of the PAI-1-mediated decrease in fibrinolysis in obesity. Figure 7E summarizes this PAI-1/LRP1/PKA/p-CREB1/PLAT pathway, as integrated with the DACH1 and Rev-Erb $\alpha$ pathways.

\section{Discussion}

Obesity has reached epidemic proportions worldwide and is a major contributor to a number of widespread chronic diseases, notably type 2 diabetes, cardiovascular disease, and nonalcoholic steatohepatitis (NASH) $(42,43)$. Among the more serious consequences of obesity, and one that contributes to cardiovascular disease, is increased risk of thrombosis $(1,3-5,8,44)$, which causes 1 in 4 deaths worldwide (45). Impaired fibrinolysis is a significant contributor to obesity-associated thrombosis $(8,14,46-50)$, but the underlying molecular mechanisms linking obesity to defects in fibrinolysis have remained largely unknown. Two critical gaps in this area of research are how obesity creates an imbalance between PAI-1 and tPA, the 2 major regulators of fibrinolysis, and the cellular source or sources of PAI-1 and tPA that contribute to this imbalance. A related gap is the mechanism and functional significance of a seemingly paradoxical observation that plasma tPA protein levels are actually increased in obese humans $(15,16)$. In this context, the major conclusions of this study are as follows: (a) hepatocytes are an important source for the increases in plasma PAI-1 and tPA in obesity; (b) hepatocyte-derived PAI-1 contributes substantially to impaired fibrinolysis in obesity; (c) hepatocyte tPA (PLAT) gene expression in obesity is regulated negatively by DACH1 and positively by PAI-1, which may represent a "compensatory" feedback pathway; (d) the net result of these opposing modes of hepatocyte PLAT regulation in obesity is an increase in plasma tPA protein, mimicking the findings in humans; and (e) although the PAI-1-mediated impairment of fibrinolysis in obesity is dominant, this impairment would be even worse without the hepatocyte PAI-1/PLAT pathway. 
A Primary human hepatocytes
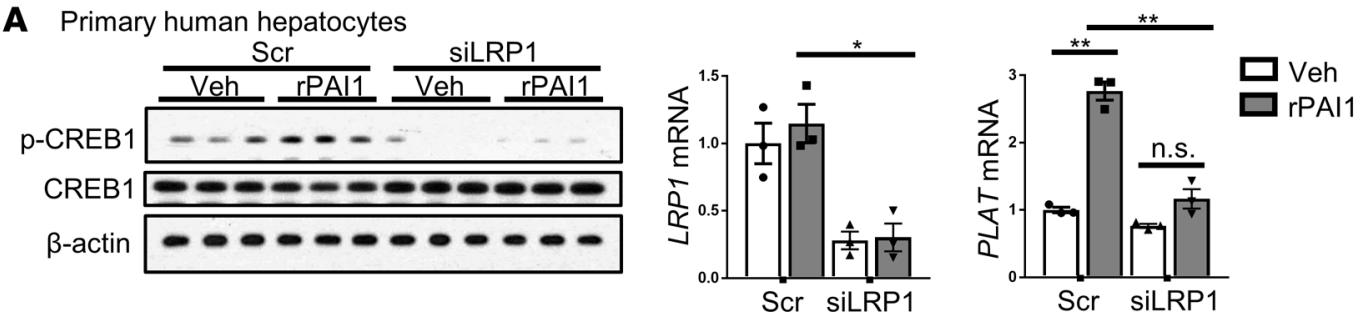
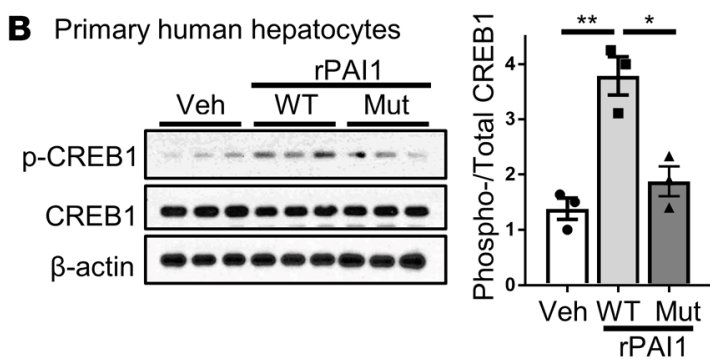
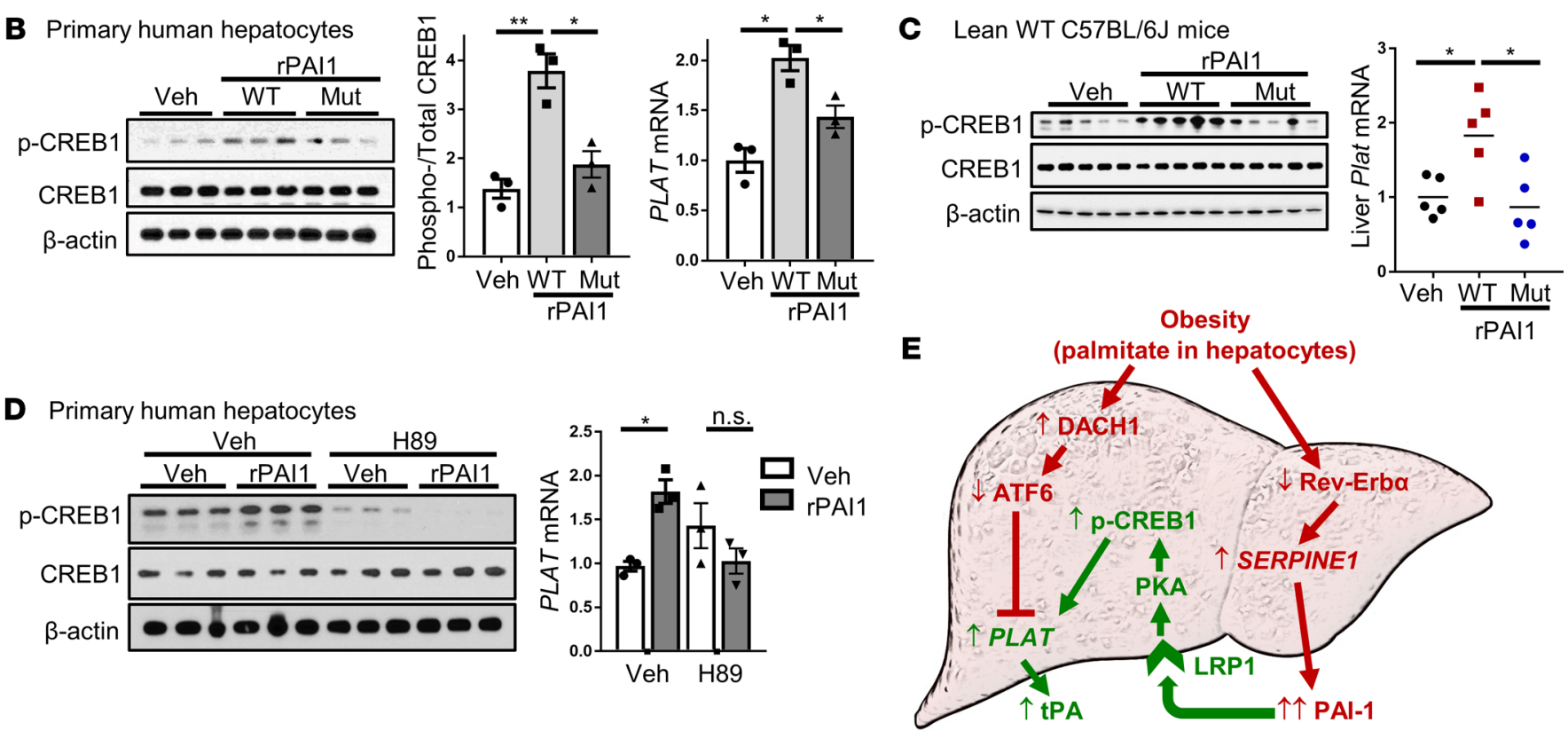

Figure 7. PAI-1 induces hepatic tPA expression through an LRP1/p-CREB1/PKA pathway. (A) Human primary hepatocytes transfected with si-LRP1 or scrambled control were treated for 8 hours with $1 \mu \mathrm{g} \mathrm{rPAI}-1 / \mathrm{mL}$ culture medium and then assayed for phosphorylated and total CREB1 and LRP1 and PLAT mRNA. (B) Human primary hepatocytes treated for 8 hours with $1 \mu \mathrm{g} / \mathrm{mL}$ WT rPAI-1 (WT) or a mutant rPAI-1 (Mut) with enzymatic activity, but lacking the LRP1-interacting heparin-binding domain, were assayed for p-CREB1 and total CREB1 and PLAT mRNA. For $\mathbf{A}$ and $\mathbf{B}$, results are represented as mean \pm SEM. $n=3$ sets of cells/group. ${ }^{*} P<0.05 ;{ }^{* *} P<0.01$, 1-way ANOVA followed by Tukey's test. (C) Lean mice were injected intravenously with $100 \mu \mathrm{g}$ WT or mutant rPAI-1 or vehicle control. After 90 minutes, livers were assayed for $p$-CREB1 and total CREB1 and Plat mRNA. $n=5$ mice/group. ${ }^{*} P<0.05,1$-way ANOVA followed by Tukey's test. (D) Human primary hepatocytes were treated for 4 hours with $10 \mu \mathrm{M}$ of PKA inhibitor H89 and then treated 4 hours with $1 \mu \mathrm{g} \mathrm{rPAI}-1 / \mathrm{mL}$. Cells were then assayed for $\mathrm{p}$-CREB1 and total CREB1 and PLAT mRNA. $n=3$ sets of cells/group. ${ }^{*} P<0.05,1$-way ANOVA followed by Tukey's test. (E) Summary schematic. Red font and arrows depict pathways that contribute to impaired fibrinolysis in obesity, and pathway in green depicts the compensatory PAI/tPA pathway. The 2 pathways that contribute to impaired fibrinolysis are increased SERPINE1 due to decreased Rev-Erb $\alpha$ and partial suppression of PLAT due to increased DACH1, which represses the PLAT inducer ATF6. The compensatory pathway is mediated by an increase in the PLAT inducer p-CREB1 downstream of a PAI-1/LRP1/PKA signaling cascade. See text for details.

Previous studies have identified adipose tissue as a major determinant of circulating PAI-1 in obesity based on PAI-1 mRNA (SERPINE1) and protein levels among various tissues $(9,14,15)$. However, another report showed that there was no arteriovenous gradient for PAI-1 protein and activity in adipose tissue in obese humans (17). Additionally, plasma PAI-1 was more closely correlated to Serpine1 mRNA in liver than in adipose tissue in obese mice (51), consistent with our findings in humans (Figure $1 \mathrm{E}$ and Supplemental Figure 1H). In our study, obesity in mice increased plasma tPA by approximately 3 -fold and plasma PAI-1 by approximately 9-fold (Supplemental Figure 1, A and B). We can estimate that approximately $60 \%$ of this plasma tPA comes from hepatocytes $(\sim 40 \%$ hepatocyte-tPA silencing led to a $25 \%$ reduced plasma tPA protein; Figure 1A) and that approximately $70 \%$ of plasma PAI-1 is hepatocyte derived (Figure 1F). Importantly, plasma tPA was reduced in the hepatocyte-PAI-1 KO obese mice (Figure 3A), indicating that the PAI-1/PLAT pathway is an important contributor to the increase in hepatocyte-derived plasma tPA in obesity. Interestingly, a SNP, rs2227667, located in the human SERPINE1 intronic region, is associated with an increase in circulating tPA levels (52), and data extracted from the GTExPortal (GTExPortal. org) suggests that this SNP may also be associated with higher SERPINE1 in human livers. If these observations are confirmed in future cohorts, they may provide genetic evidence for the PAI-1/ tPA pathway described herein.

In addition to affecting systemic fibrinolysis in obesity, regulation of fibrinolysis by hepatocytes may have other important implications in both disease and normal physiology. For example, obesity markedly increases the risk for chronic liver disease by promoting the development of NASH (53), and patients with 
liver disease are susceptible to both local thrombosis $(54,55)$, i.e., in the portal vein, and to systemic thrombosis (56-59). In terms of possible relevance to normal physiology, acute fluctuations in hepatic PAI-1 expression, e.g., in response to a meal or during infection (60-62), might require a compensatory increase in tPA to prevent impaired fibrinolysis. This response may then become compromised by disease-related processes, as exemplified by the elevation of DACH1 in hepatocytes in obesity, which decreases tPA expression and thereby limits the compensatory response. Interestingly, we showed previously that increased hepatocyte DACH1 in obesity also promotes excessive hepatic glucose production (32). Thus, hepatocyte DACH1 emerges as an important link between obesity-associated metabolic stress and impaired fibrinolysis. Accordingly, suppression of DACH1 using hepatocyte-targeted siRNA strategies, a modality that has been approved for use in humans (63), may provide an integrated approach to the problem of hepatocyte-mediated perturbations in metabolic disease.

The hepatocyte PAI-1/tPA pathway described here is unique when considering the pathophysiology of clotting disorders in obesity because hepatocytes can be considered to be at the intersection of sensing obesity-induced metabolic stress and regulating fibrinolysis. Thus, understanding this pathway may inform new strategies for improving basal fibrinolysis in obese subjects before an injury occurs. However, these strategies would have to take into account other perturbations associated with obesity, notably those related to coagulation and platelet function (64-69), which also contribute to the high risk of thrombotic disorders in the obese population (50, 70-72). Accordingly, the findings herein will likely have to be integrated with understanding of these other processes to fully define the pathophysiology of thrombotic disease in obesity and to conceive new therapies.

\section{Methods}

Mouse models. WT mice used for silencing hepatocyte tPA (Figure 1A) and expressing hepatocyte Rev-Erb $\alpha$ (Figure 4C) were purchased from The Jackson Laboratory (JAX) as age-controlled lean and DIO mice (catalog 000664 and 380050, respectively). JAX DIO mice are available only as male mice. Both lean and obese mice were purchased at the age of 15 weeks and maintained with the same chow or DIO diet (Research Diets, catalog 12492) after transferring to the animal facility at Columbia University Irving Medical Center. After 1 to 2 weeks of adjustment to the new environment, AAV8 viruses were injected into the DIO mice when they were 17 weeks old. Experiments were started 3 weeks after the AAV8 injection. The mice were euthanized at the age of 22 to 23 weeks, so that the total time of DIO diet feeding was 4 months. The AAV8 constructs used were AAV8-H1-shPlat (13), to silence hepatocyte Plat, and AAV8-TBG-Nr1d1 (73), to express hepatocyte Rev-Erba. AAV8 vectors were delivered at $1 \times 10^{11}$ genome copies/mouse. For AAV8TBG-Nr1d1, the dose we used was $10 \%$ of the viral dose used in the original publication (73) to avoid excess expression. The AAV8TBG-Nr1d1 vector was provided by Mitchell Lazar (University of Pennsylvania, Philadelphia, Pennsylvania, USA). Control mice were age-matched WT mice injected with AAV8-H1-LacZ and kept in the same animal facility for the same period.
The Serpine $1^{f / / l}$ mice (Figure 1, F-I), Dach $1^{f / / f l}$ mice (Figure 2A), and $\mathrm{Creb}^{\mathrm{fl} / \mathrm{fl}}$ mice (Figure 6) were housed in the animal facility at Columbia University Irving Medical Center from birth. They were placed and maintained on a DIO diet (Research Diets, catalog 12492) at 6 weeks of age. The same DIO protocol with the same DIO diet, followed by the same AAV8 viral injection timing protocol, was used as above. Both male and female mice were used in these cohorts. Specifically, Serpine $1^{f / / f l}$ mice were generated and crossed onto the C57BL/6J background, as previously described (28). The mice were injected intravenously with AAV8-TBG-Cre at 4 months of age to delete PAI- 1 in hepatocytes (13, 29-32, 74) (HC-PAI-1-KO mice). Control mice were Serpine $1^{f / f l}$ mice injected with the AAV8-TBG-LacZ virus. Dach ${ }^{f / f l}$ mice were generated as previously described (75) and crossed onto the C57BL/6J background. The mice were injected intravenously with AAV8-TBG-Cre at 4 months of age to deplete DACH1 in hepatocytes $(13,29-32,74)$ (HC-DACH1-KO mice). Control mice were Dach $\mathbf{f}^{f / f l}$ mice injected with the AAV8-TBG-LacZ virus. The Dach $1^{f / f l}$ mice were provided by Richard Pestell (Baruch S. Blumberg Institute, Doylestown, Pennsylvania, USA, and Nanyang Technological University, Singapore). AAV8 vectors were delivered at $1 \times 10^{11}$ genome copies/mouse, and experiments were commenced 3 to 6 weeks later. Creb $1^{f / f l}$ mice were generated as previously described (76) and crossed onto the C57BL/6J background. The mice were injected intravenously with AAV8-TBG-Cre at 4 months of age to delete CREB1 in hepatocytes $(13,29-32,74)$ (HC-CREB1-KO mice). Control mice were $\mathrm{Creb}^{\mathrm{fl} / \mathrm{fl}}$ mice injected with the AAV8-TBG-LacZ virus. The Creb1 ${ }^{f / l}$ mice were provided by Eric J. Nestler (Icahn School of Medicine at Mount Sinai, New York, New York, USA).

For all experiments, mice were maintained on a 12-hour light/12hour dark cycle with free access to normal chow or the DIO diet and water. Mice of the same age and similar weight were randomly assigned to experimental and control groups. Body weight was maintained throughout. Data for 5 weeks after AAV8 injection are shown in Supplemental Figure 6.

Mouse thrombosis assays. Carotid artery thrombosis was induced by either $\mathrm{FeCl}_{3}$-induced injury or by rose bengal/laser-induced photochemical injury, as previously described $(77,78)$. Briefly, mice were anesthetized with isoflurane and placed on a thermo-controlled blanket $\left(37^{\circ} \mathrm{C}\right)$, followed by surgical exposure of the carotid artery. For the $\mathrm{FeCl}_{3}$ procedure, a filter paper soaked in $10 \% \mathrm{FeCl}_{3}$ was applied to the artery for 3 minutes, followed by rinsing with normal saline. Blood flow was monitored with an ultrasound flow probe (Transonics) and recorded by LabChart software (ADInstruments). The time to total occlusion was defined as the interval between the application of $\mathrm{FeCl}_{3}$ and stable occlusion of the artery, defined as 0 blood flow for 3 minutes (77). For the rose bengal/laser procedure, $50 \mathrm{mg} / \mathrm{kg}$ of rose bengal in $0.15 \mathrm{~mL}$ of $0.9 \%$ saline was injected through the tail vein. A $540-\mathrm{nm}$ wavelength laser light source (Melles Griot) held $6 \mathrm{~cm}$ away from the carotid artery was switched on to initiate photochemical injury, and then blood flow in the artery was monitored continuously (78). The time to total occlusion was defined as the interval between application of the laser and stable occlusion of the artery, with 0 blood flow for 10 minutes. After the procedure, the mice were immediately euthanized.

Mouse tail-bleeding assay. Mice were anesthetized with isoflurane and positioned horizontally on a platform that allowed the tail to descend approximately $2 \mathrm{~cm}$ from the top of the platform. A distal segment of the tail was transected with a no. 11 surgical scalpel to induce wounds of approximately 2 -mm diameter. Bleeding time was monitored by gen- 
tly dabbing the tail tip on Whatman paper at 10-second intervals until the cessation of bleeding (79). The time to stable cessation of bleeding was defined as the time interval between the tail incision and cessation of bleeding, with no evidence of rebleeding for 60 seconds. Bleeding exceeding 15 minutes was stopped by applying pressure.

Plasma collection and analyses. Blood obtained by cardiac puncture into $10 \%$ volume of sodium citrate $(3.8 \%, \mathrm{w} / \mathrm{v})$ was centrifuged for 15 minutes at $2300 \mathrm{~g}$, and plasma was carefully collected from the supernatant fraction. Plasma samples were divided into aliquots, snap-frozen, and stored at $-80^{\circ} \mathrm{C}$ until analyses. Plasma total antigen levels of tPA, PAI-1, and A2AP were measured by ELISA using kits according to the manufacturer's instructions (catalog numbers are listed in Supplemental Methods). Plasma PAI-1-free tPA was measured by ELISA by first capturing the free tPA on a surface coated with PAI-1 antigen, enabling detection of only the functional, PAI-1-free form of tPA. tPA enzymatic activity in plasma or tissue lysates was assayed by chromatographically measuring the release of para-nitroaniline (pNA) chromophore from a plasmin-specific synthetic substrate (Abcam, ab108905). Results were recorded and analyzed by VersaMax Microplate Reader and SoftMax Pro software (Molecular Devices, Thermo Fisher Scientific).

Euglobulin clot-lysis time. A total of $50 \mu \mathrm{L}$ of citrated mouse plasma was resuspended in $900 \mu \mathrm{L} 0.017 \%$ acetic acid, placed on ice for 20 minutes, and then centrifuged for 20 minutes at $2000 \mathrm{~g}$ at $4^{\circ} \mathrm{C}$. After careful removal of the supernatant fraction, each pellet (euglobulin fraction) was resuspended in $55 \mu \mathrm{L}$ sodium borate $/ \mathrm{NaCl}$ ( $\mathrm{pH} 9.0$ ) and transferred to a single well on a flat-bottom 96-well microtiter assay plate; $50 \mu \mathrm{L}$ of $25 \mathrm{mM} \mathrm{CaCl}_{2}$ was added to each well, and then the absorbance was recorded at $405 \mathrm{~nm}$ every 10 minutes, with 3-second shakes before each reading, at room temperature for 16 hours. Clot-lysis time was calculated as the time to achieve $50 \%$ of clot lysis (half-lysis time) (80).

Human and mouse primary hepatocyte experiments. Human primary hepatocytes were obtained from the Liver Tissue Cell Distribution System at the University of Pittsburgh (Pittsburgh, Pennsylvania, USA). Primary mouse hepatocytes were isolated from 10-week-old WT C57BL/6J mice, as described previously $(32,81)$. All cells were cultured in DMEM containing 10\% FBS and then transfected with siRNAs described in the figure legends. The cells were then cultured in serum-free medium until they were harvested into RIPA buffer (Thermo Fisher Scientific, catalog 89900) with Halt Protease and Phosphatase Inhibitor Cocktail (Thermo Fisher Scientific, catalog 78444) for immunoblotting or into RNA lysis buffer (QIAGEN, catalog 79216) for mRNA quantification. Culture media were collected, snap-frozen in liquid nitrogen, and stored at $-80^{\circ} \mathrm{C}$ until processing.

Human liver specimens. Human liver specimens were acquired from patients undergoing bariatric surgery or clinically indicated laparoscopic procedures at the New York Presbyterian Hospital, Columbia University Irving Medical Center (ref. 32, Figure 1E, Figure 2B, Figure 3D, and Supplemental Figure 3C). The liver specimens were from intraoperative needle biopsies and were frozen immediately in liquid nitrogen and stored at $-80^{\circ} \mathrm{C}$ until subsequent analyses. The clinical characteristics of the individual donors appear in Supplemental Tables 1 and 2. We also received human liver specimens from the Liver Tissue Cell Distribution System at the University of Minnesota (Minneapolis, Minnesota, USA) (ref. 13 and Figure 2B). The specimens were collected postmortem on the date of liver transplantation and preserved as frozen samples.
Statistics. All results are presented as mean \pm SEM. $P$ values were calculated using 2-tailed Student's $t$ test for data that passed the normality test or the Mann-Whitney rank-sum $U$ test for data that were not normally distributed. One-way ANOVA with post hoc Tukey's test was used to evaluate differences among groups when 3 or more groups were analyzed.

Study approval. All mouse experiments were conducted with the approval of the IACUC of Columbia University Medical Center. The use of human cells and specimens in this study was approved by the IRB at the Columbia University Irving Medical Center. All participants provided written informed consent.

\section{Author contributions}

$\mathrm{ZZ}$ and IT designed the research. ZZ, KN, SG, XW, and ST conducted the research. RML provided critical reagents related to PAI-1. ST, MB, BS, and AK organized patient recruitment and human liver sample collection. LO provided critical reagents and advice related to DACH1. ZZ, KN, SG, LO, JAL, and IT analyzed the data. ZZ, SG, JAL, and IT wrote the manuscript. All authors read and commented on the manuscript.

\section{Acknowledgments}

We thank Arif Yurdagul Jr., Bishuang Cai, Nan Wang, Alan Tall, and Henry Ginsberg (Columbia University Irving Medical Center), Lalitha Nayak and Mukesh Jain (Case Western Reserve University) and Katherine Hajjar (Weill Cornell Medicine) for helpful discussions; Richard Pestell (Baruch S. Blumberg Institute and Nanyang Technological University) for providing the Dach $1^{f / f l}$ mice; Eric J. Nestler (Icahn School of Medicine at Mount Sinai) for providing the $\mathrm{Creb}^{f l / f l}$ mice; Mitchell Lazar (University of Pennsylvania) for providing the AAV8-TBG-Nr1d1 vector; George Kuriakose (Columbia University Irving Medical Center) for technical support; and Marion Namenwirth and Abigail Knoble (University of Minnesota) and Nicole Martik (University of Pittsburgh) for arranging and providing human liver samples and primary hepatocytes, respectively. ZZ was funded by a Berrie Scholar Post-Doctoral Fellowship Award from the Russell Berrie Foundation, an NIH postdoctoral fellow T32 institutional training grant (HL007343), a Fellow Scholar Award from the American Society of Hematology, and a Career Development Award from the American Heart Association (19CDA34660043); KN by a Medical Scientist Training Scholarship from the University of Tokyo; SG by a Summer Research Institute Scholarship from Barnard College and by a Fellow Scholar Award from the American Society of Hematology (to ZZ); XW by an American Liver Foundation Liver Scholar Award; LO by NIH grant DK106045 and a Pilot and Feasibility Grant from the Columbia University Diabetes Research Center (DK063608); RML by NIH grant HL114470; JAL by NIH grant HL145262; and IT by NIH grant HL087123. Human liver samples and primary hepatocytes were obtained from the Liver Tissue Cell Distribution System (University of Minnesota and the University of Pittsburgh), which was funded by NIH contract HHSN276201200017C.

Address correspondence to: Ze Zheng or Ira Tabas, Department of Medicine, Columbia University Irving Medical Center, 630 West 168th Street, New York, NY 10032, USA. Phone: 212-305-5669; Email: zz2391@columbia.edu (ZZ). Phone: 212-305-9430; Email: iat1@columbia.edu (IT). 
1. Wolk R, Berger P, Lennon RJ, Brilakis ES, Somers VK. Body mass index: a risk factor for unstable angina and myocardial infarction in patients with angiographically confirmed coronary artery disease. Circulation. 2003;108(18):2206-2211.

2. Hubert HB, Feinleib M, McNamara PM, Castelli WP. Obesity as an independent risk factor for cardiovascular disease: a 26-year follow-up of participants in the Framingham Heart Study. Circulation . 1983;67(5):968-977.

3. Suk SH, et al. Abdominal obesity and risk of ischemic stroke: the Northern Manhattan Stroke Study. Stroke. 2003;34(7):1586-1592.

4. Yang G, De Staercke C, Hooper WC. The effects of obesity on venous thromboembolism: A review. Open J Prev Med. 2012;2(4):499-509.

5. Stein PD, Beemath A, Olson RE. Obesity as a risk factor in venous thromboembolism. Am JMed. 2005;118(9):978-980.

6. Stein PD, Matta F, Goldman J. Obesity and pulmonary embolism: the mounting evidence of risk and the mortality paradox. Thromb Res. 2011;128(6):518-523.

7. Nicol KK, Shelton BJ, Knovich MA, Owen J. Overweight individuals are at increased risk for thrombotic thrombocytopenic purpura. Am J Hematol. 2003;74(3):170-174.

8. Blokhin IO, Lentz SR. Mechanisms of thrombosis in obesity. Curr Opin Hematol. 2013;20(5):437-444.

9. Lentz SR. Thrombosis in the setting of obesity or inflammatory bowel disease. Blood. 2016;128(20):2388-2394.

10. Cesarman-Maus G, Hajjar KA. Molecular mechanisms of fibrinolysis. Br J Haematol. 2005;129(3):307-321.

11. Medved L, Nieuwenhuizen W. Molecular mechanisms of initiation of fibrinolysis by fibrin. Thromb Haemost. 2003;89(3):409-419.

12. Wun TC, Capuano A. Initiation and regulation of fibrinolysis in human plasma at the plasminogen activator level. Blood. 1987;69(5):1354-1362.

13. Zheng Z, et al. An ATF6-tPA pathway in hepatocytes contributes to systemic fibrinolysis and is repressed by DACH1. Blood. 2019;133(7):743-753.

14. Skurk T, Hauner H. Obesity and impaired fibrinolysis: role of adipose production of plasminogen activator inhibitor-1. Int JObes Relat Metab Disord. 2004;28(11):1357-1364.

15. Janand-Delenne B, Chagnaud C, Raccah D, Alessi MC, Juhan-Vague I, Vague P. Visceral fat as a main determinant of plasminogen activator inhibitor 1 level in women. Int J Obes Relat Metab Disord. 1998;22(4):312-317.

16. Ladenvall P, Nilsson S, Jood K, Rosengren A, Blomstrand C, Jern C. Genetic variation at the human tissue-type plasminogen activator (tPA) locus: haplotypes and analysis of association to plasma levels of tPA. Eur J Hum Genet. 2003;11(8):603-610.

17. Yudkin JS, Coppack SW, Bulmer K, Rawesh A, Mohamed-Ali V. Lack of evidence for secretion of plasminogen activator inhibitor-1 by human subcutaneous adipose tissue in vivo. Thromb Res. 1999;96(1):1-9.

18. Simpson AJ, Booth NA, Moore NR, Bennett B. Distribution of plasminogen activator inhibitor (PAI-1) in tissues. J Clin Pathol. 1991;44(2):139-143.

19. Lee SM, Dorotea D, Jung I, Nakabayashi T, Miya- ta T, Ha H. TM5441, a plasminogen activator inhibitor-1 inhibitor, protects against high fat diet-induced non-alcoholic fatty liver disease. Oncotarget. 2017;8(52):89746-89760.

20. Kietzmann T, Roth U, Jungermann K. Induction of the plasminogen activator inhibitor-1 gene expression by mild hypoxia via a hypoxia response element binding the hypoxia-inducible factor-1 in rat hepatocytes. Blood. 1999;94(12):4177-4185.

21. Kietzmann T, Jungermann K, Görlach A. Regulation of the hypoxia-dependent plasminogen activator inhibitor 1 expression by MAP kinases. Thromb Haemost. 2003;89(4):666-673.

22. Chandler WL, et al. Prothrombotic coagulation abnormalities preceding the hemolytic-uremic syndrome. N Engl JMed. 2002;346(1):23-32.

23. Agren A, Jörneskog G, Elgue G, Henriksson $\mathrm{P}$, Wallen $\mathrm{H}$, Wiman B. Increased incorporation of antiplasmin into the fibrin network in patients with type 1 diabetes. Diabetes Care. 2014;37(7):2007-2014.

24. Steffanina A, Proietti L, Antonaglia C, Palange P, Angelici E, Canipari R. The plasminogen system and transforming growth factor- $\beta$ in subjects with obstructive sleep apnea syndrome: effects of CPAP treatment. Respir Care. 2015;60(11):1643-1651.

25. Chandler WL, Alessi MC, Aillaud MF, Henderson P, Vague P, Juhan-Vague I. Clearance of tissue plasminogen activator (TPA) and TPA/plasminogen activator inhibitor type 1 (PAI-1) complex: relationship to elevated TPA antigen in patients with high PAI-1 activity levels. Circulation. 1997;96(3):761-768.

26. Yarmolinsky J, Bordin Barbieri N, Weinmann $\mathrm{T}$, Ziegelmann PK, Duncan BB, Inês Schmidt M. Plasminogen activator inhibitor-1 and type 2 diabetes: a systematic review and meta-analysis of observational studies. Sci Rep. 2016;6:17714.

27. Eliasson MC, Jansson JH, Lindahl B, Stegmayr B. High levels of tissue plasminogen activator (tPA) antigen precede the development of type 2 diabetes in a longitudinal population study. The Northern Sweden MONICA study. Cardiovasc Diabetol. 2003;2:19.

28. Jiang C, et al. Serpine 1 induces alveolar type II cell senescence through activating p53-p21-Rb pathway in fibrotic lung disease. Aging Cell. 2017;16(5):1114-1124.

29. Ghorpade DS, et al. Hepatocyte-secreted DPP4 in obesity promotes adipose inflammation and insulin resistance. Nature. 2018;555(7698):673-677.

30. Wang X, et al. Hepatocyte TAZ/WWTR1 promotes inflammation and fibrosis in nonalcoholic steatohepatitis. Cell Metab. 2016;24(6):848-862.

31. Lisowski L, et al. Selection and evaluation of clinically relevant AAV variants in a xenograft liver model. Nature. 2014;506(7488):382-386.

32. Ozcan L, et al. Hepatocyte DACH1 is increased in obesity via nuclear exclusion of HDAC4 and promotes hepatic insulin resistance. Cell Rep. 2016;15(10):2214-2225.

33. Matsuzaka T, et al. Crucial role of a long-chain fatty acid elongase, Elovl6, in obesity-induced insulin resistance. Nat Med. 2007;13(10):1193-1202.

34. Wang J, Yin L, Lazar MA. The orphan nuclear receptor Rev-erb alpha regulates circadian expression of plasminogen activator inhibitor type 1. J Biol Chem. 2006;281(45):33842-33848.

35. Vieira E, et al. Altered clock gene expression in obese visceral adipose tissue is associated with metabolic syndrome. PLoS One. 2014;9(11):e111678.

36. Costa M, Shen Y, Maurer F, Medcalf RL. Transcriptional regulation of the tissue-type plasminogen-activator gene in human endothelial cells: identification of nuclear factors that recognise functional elements in the tissue-type plasminogen-activator gene promoter. Eur J Biochem. 1998;258(1):123-131.

37. Herzig S, et al. CREB regulates hepatic gluconeogenesis through the coactivator PGC-1. Nature. 2001;413(6852):179-183.

38. Zhu Y, Hui DY. Apolipoprotein E binding to low density lipoprotein receptor-related protein-1 inhibits cell migration via activation of cAMP-dependent protein kinase A. J Biol Chem. 2003;278(38):36257-36263.

39. Kandel ER. The molecular biology of memory: CAMP, PKA, CRE, CREB-1, CREB-2, and CPEB. Mol Brain. 2012;5:14.

40. Nakajima C, et al. Low density lipoprotein receptor-related protein 1 (LRP1) modulates $\mathrm{N}$-methyl-D-aspartate (NMDA) receptor-dependent intracellular signaling and NMDA-induced regulation of postsynaptic protein complexes. J Biol Chem . 2013;288(30):21909-21923.

41. Ikoma-Seki K, et al. Role of LRP1 and ERK and cAMP signaling pathways in lactoferrin-induced lipolysis in mature rat adipocytes. PLoS One. 2015;10(10):e0141378.

42. Björntorp P. Obesity: a chronic disease with alarming prevalence and consequences. J Intern Med.1998;244(4):267-269.

43. Kearns K, Dee A, Fitzgerald AP, Doherty E, Perry IJ. Chronic disease burden associated with overweight and obesity in Ireland: the effects of a small BMI reduction at population level. BMC Public Health. 2014;14:143.

44. Must A, McKeown NM. The disease burden associated with overweight and obesity. In: De Groot LJ, et al., eds. Endotext. South Dartmouth, Massachusetts, USA; 2000.

45. ISTH Steering Committee for World Thrombosis Day. Thrombosis: a major contributor to global disease burden. Thromb Res. 2014;134(5):931-938.

46. Lijnen HR. Role of fibrinolysis in obesity and thrombosis. Thromb Res. 2009;123 Suppl 4:S46-S49.

47. Juhan-Vague I, et al. Relationships between plasma insulin triglyceride, body mass index, and plasminogen activator inhibitor 1. Diabete Metab. 1987;13(3 Pt 2):331-336.

48. Landin $\mathrm{K}$, et al. Abdominal obesity is associated with an impaired fibrinolytic activity and elevated plasminogen activator inhibitor-1. Metab Clin Exp. 1990;39(10):1044-1048.

49. McGill JB, Schneider DJ, Arfken CL, Lucore CL, Sobel BE. Factors responsible for impaired fibrinolysis in obese subjects and NIDDM patients. Diabetes. 1994;43(1):104-109.

50. Potter van Loon BJ, Kluft C, Radder JK, Blankenstein MA, Meinders AE. The cardiovascular risk factor plasminogen activator inhibitor type 1 is related to insulin resistance. Metab Clin Exp. 1993;42(8):945-949.

51. Alessi MC, et al. Plasma PAI-1 levels are more 
strongly related to liver steatosis than to adipose tissue accumulation. Arterioscler Thromb Vasc Biol. 2003;23(7):1262-1268.

52. Huang J, et al. Genome-wide association study for circulating tissue plasminogen activator levels and functional follow-up implicates endothelial STXBP5 and STX2. Arterioscler Thromb Vasc Biol. 2014;34(5):1093-1101.

53. Sarwar R, Pierce N, Koppe S. Obesity and nonalcoholic fatty liver disease: current perspectives. Diabetes Metab Syndr Obes. 2018;11:533-542.

54. Hernández-Conde $\mathrm{M}$, et al. Visceral fat is associated with cirrhotic portal vein thrombosis. Expert Rev Gastroenterol Hepatol. 2019;13(10):1017-1022.

55. Ayala R, et al. Obesity is an independent risk factor for pre-transplant portal vein thrombosis in liver recipients. BMC Gastroenterol. 2012;12:114.

56. Lisman T, Leebeek FW, de Groot PG. Haemostatic abnormalities in patients with liver disease. J Hepatol. 2002;37(2):280-287.

57. Bennani-Baiti N, Daw HA. Primary hyperfibrinolysis in liver disease: a critical review. Clin $A d v$ Hematol Oncol. 2011;9(3):250-252.

58. Leebeek FW, Rijken DC. The fibrinolytic status in liver diseases. Semin Thromb Hemost. 2015;41(5):474-480.

59. Luyendyk JP, Schoenecker JG, Flick MJ. The multifaceted role of fibrinogen in tissue injury and inflammation. Blood. 2019;133(6):511-520.

60. Byrne CD, Wareham NJ, Martensz ND, Humphries SE, Metcalfe JC, Grainger DJ. Increased PAI activity and PAI- 1 antigen occurring with an oral fat load: associations with PAI-1 genotype and plasma active TGF-beta levels. Atherosclerosis. 1998;140(1):45-53.

61. Chang ML, Lin YS, Pao LH, Huang HC, Chiu CT. Link between plasminogen activator inhibitor-1 and cardiovascular risk in chronic hepatitis $C$ after viral clearance. Sci Rep. 2017;7:42503.

62. de Boer JP, et al. PAI-1 synthesis in the human hepatoma cell line HepG2 is increased by cytokines--evidence that the liver contributes to acute phase behaviour of PAI-1. Thromb Haemost 1991;65(2):181-185.

63. Adams D, et al. Patisiran, an RNAi therapeutic, for hereditary transthyretin amyloidosis. N EnglJ Med. 2018;379(1):11-21.

64. Samad F, Ruf W. Inflammation, obesity, and thrombosis. Blood. 2013;122(20):3415-3422.

65. Ay L, et al. Thrombin generation in morbid obesity: significant reduction after weight loss. J Thromb Haemost. 2010;8(4):759-765.

66. Kopp CW, et al. Weight loss reduces tissue factor in morbidly obese patients. Obes Res. 2003;11(8):950-956.

67. Tschoner A, et al. Plasminogen activator inhibitor 1 and visceral obesity during pronounced weight loss after bariatric surgery. Nutr Metab Cardiovasc Dis. 2012;22(4):340-346.

68. Russo I, et al. In central obesity, weight loss restores platelet sensitivity to nitric oxide and prostacyclin. Obesity (Silver Spring). 2010;18(4):788-797.

69. Miszta A, et al. A high-fat diet delays plasmin generation in a thrombomodulin-dependent manner in mice. Blood. 2020;135(19):1704-1717.

70. Stine JG, Shah NL, Argo CK, Pelletier SJ, Caldwell SH, Northup PG. Increased risk of portal vein thrombosis in patients with cirrhosis due to nonalcoholic steatohepatitis. Liver Transpl. 2015;21(8):1016-1021.

71. Stine JG, et al. Increased risk of venous thromboembolism in hospitalized patients with cirrhosis due to non-alcoholic steatohepatitis. Clin Transl
Gastroenterol. 2018;9(3):140.

72. Vazzana N, Ranalli P, Cuccurullo C, Davì G. Diabetes mellitus and thrombosis. Thromb Res. 2012;129(3):371-377.

73. Fang B, et al. Circadian enhancers coordinate multiple phases of rhythmic gene transcription in vivo. Cell. 2014;159(5):1140-1152.

74. Zheng Z, et al. An ATF6-tPA pathway in hepatocytes contributes to systemic fibrinolysis and is repressed by DACH1. Blood. 2019;133(7):743-753.

75. Chen K, et al. The endogenous cell-fate factor dachshund restrains prostate epithelial cell migration via repression of cytokine secretion via a cxcl signaling module. Cancer Res. 2015;75(10):1992-2004.

76. Covington HE, et al. A role for repressive histone methylation in cocaine-induced vulnerability to stress. Neuron. 2011;71(4):656-670.

77. Wang $\mathrm{W}$, et al. LNK/SH2B3 loss of function promotes atherosclerosis and thrombosis. Circ Res. 2016;119(6):e91-e103.

78. Nayak L, Shi H, Atkins GB, Lin Z, Schmaier AH, Jain MK. The thromboprotective effect of bortezomib is dependent on the transcription factor Kruppel-like factor 2 (KLF2). Blood. 2014;123(24):3828-3831.

79. Kung SH, et al. Human factor IX corrects the bleeding diathesis of mice with hemophilia B. Blood.1998;91(3):784-790.

80. Marcos-Contreras OA, et al. Hyperfibrinolysis increases blood-brain barrier permeability by a plasmin- and bradykinin-dependent mechanism. Blood. 2016;128(20):2423-2434.

81. Bellahcene M, et al. Activation of p38 mitogen-activated protein kinase contributes to the early cardiodepressant action of tumor necrosis factor. J Am Coll Cardiol. 2006;48(3):545-555. 